\section{Amino/aromatic interactions}

SIR - Rodham et al. ${ }^{1}$ detailed both experimental and theoretical investigations of the structure of the ammonia/benzene complex. Following the identification of an amino/aromatic hydrogen bond in $\mathrm{SH} 2$ domain/peptide binding by Waksman et $a l .^{2}$, there has been a resurgence of interest in such interactions in proteins. We believe that this interest needs to be accompanied by an understanding of the rarity and relatively weak energies of these interactions compared with other kinds. Before Levitt and Perutz ${ }^{3}$ first

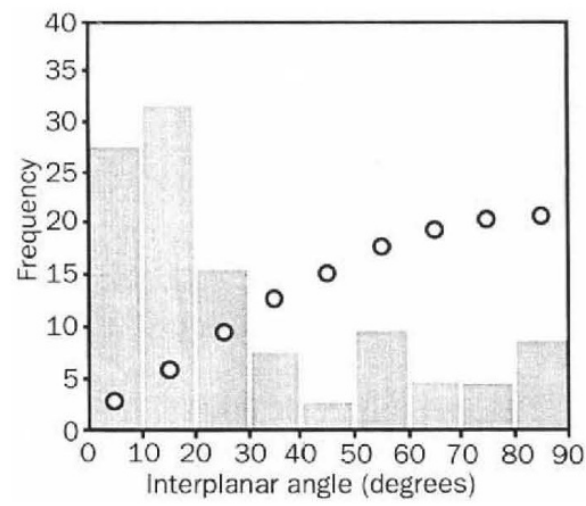

Distribution of the interplanar angle between $s p^{2}$ nitrogen and an aromatic ring for 116 amino/aromatic interactions in which the nitrogen lies above the ring face. This is defined by requiring the nitrogen to be within $20^{\circ}$ of being directly above either a ring carbon or the ring centre, and no more than $3.8 \AA$ away from the same site. The dataset includes both sideand main-chain nitrogens and both phenylalanine and tyrosine rings. Seven interactions involving lysine side-chains are, however, excluded from this plot as the interplanar angle is undefined, these nitrogens being $s p^{3}$ hybridized. Our definition of an amino/aromatic hydrogen bond requires an 'above ring' interaction with $\mathrm{N}-\mathrm{H}^{\cdots} \mathrm{C}<120^{\circ}$ and an interplanar angle (not applicable to lysine sidechains) $\geqslant 30^{\circ}$. All 'above ring' interactions with interplanar angles $<30^{\circ}$ are classified as stacked. Circles represent the sinusoidal expected distribution.

suggested that amino/aromatic hydrogen bonds might be important in proteins, Legon and Millen $^{4}$ had already established that proton donor/ $\pi$-electron interactions are, at least for small molecules, observed if, and only if, conventional hydrogen bonding to lone-pair acceptors is not possible. Indeed, our own energy calculations reinforce the view that

1. Rodham, D. A. et al Nature 362, 735-737 (1993)

1. Rodham, D. A. et al. Nature 362, 735-737 (1993)

2. Waksman, G. etal. Nature 358, 646-653(1992). (1988)

4. Legon, A. C. \& Millen, D. J. Acc. chem. Res. 20, 39-46 (1987)

5. Bernstein, F. C. etal. J. molec. Biol. 112, 535-542 (1977)

6. Suzuki. S. etal. Science 257, 942-945 (1992)

7. Mitchell, J. B. O. \& Price, S. L. Chem. phys. Lett. 154 267-272 (1989). such an interaction is significantly weaker than a normal hydrogen bond, but preferable to leaving a potential hydrogenbonding proton totally unfulfilled.

We have studied various amino/ aromatic interactions, especially those 'above ring' cases where any nitrogen atom lies above the phenylalanine or tyrosine rings (requiring an atom-atom contact distance of less than $3.8 \AA$ ). We used 58 non-homologous high-resolution Brookhaven ${ }^{5}$ protein structures and added inferred hydrogen positions to the structures, enabling us to search for all amino/aromatic hydrogen bonds. However, we found that the majority of 'above ring' amino/aromatic interactions involve stacking of the planar $s p^{2}$ nitrogens above their aromatic partners; only a few are hydrogen bonded. Out of 1,171 residue/residue interactions, 123 are 'above ring'; of these, 36 are hydrogen bonded, 76 stacked and 11 neither (see figure). Thus only 3 per cent of all amino/ aromatic interactions found are hydrogen bonds.

Our $a b$ initio calculations find that the isolated dimer interaction energy, when taken alone, favours hydrogen-bonded conformations over stacked ones. Within proteins, in contrast, the stacked geometry is generally favoured as it allows all potential hydrogen-bonding protons to interact with conventional acceptors,

\section{$\mathrm{X}$-rays from a gamma-ray repeater?}

SIR - Kulkarni and Frail ${ }^{1}$ pointed out the occurrence within the localization box of the soft $\gamma$-ray repeater SGR1806-20 (refs $2,3)$ of the 'amorphous radio nebula' G10.1 - 0.3 from Green's supernova remnant (SNR) catalogue ${ }^{4}$. They argue that all SGRs are probably young neutron stars still embedded in the remnants of the supernovae that gave rise to them, and that the characteristic signatures of SNRs and rotating, magnetized neutron stars should therefore be observable. Further radio observations of $\mathrm{G} 10.1-0.3$ by Kulkarni et al.$^{5}$ reveal a compact nebula with its centroid at coordinates (1950) 18 h 05 $\min 41.76 \mathrm{~s},-20^{\circ} 25^{\prime} 13^{\prime \prime}$ superimposed on an extended plateau of emission. This structure suggests a pulsar-powered SNR - a plerion - which, by analogy with the Vela pulsar ${ }^{6}$, could be a detectable X-ray source.

I observed the field containing the localization box of SGR1806-20 (including the smaller, revised localization box cited as a personal communication in ref. 1) with the position-sensitive proportional counter of the Rosat X-ray telescope in April 1993. The observation achieved $9,729 \mathrm{~s}$ at the centre of the $2^{\circ}$ field and whether from protein or solvent. Thus the forming of conventional hydrogen bonds generally dominates amino/aromatic interactions, as the relative paucity of amino/aromatic hydrogen bonds shows.

Where amino/aromatic hydrogen bonding is indicated, we usually find that one proton points roughly towards a ring carbon; the second proton on an amino group points away, apparently in accordance with the monodentate nature of the ammonia/benzene $^{1}$ and water/benzene ${ }^{6}$ structures. But as this second proton generally forms a conventional hydrogen bond of its own, the geometry is in fact likely to be a compromise between optimizing the two donor/acceptor interactions ${ }^{7}$.

John B. O. Mitchell, C. Lilian Nandi, Shamimara Ali, Ian K. McDonald, Janet $M$. Thornton

Biomolecular Structure and

Modelling Unit,

Department of Biochemistry and Molecular Biology,

University College London,

Gower Street, London WC1E 6BT, UK

Sarah L. Price

Department of Chemistry,

University College London,

London WC1HOAJ, UK

Juswinder Singh

Parke-Davis Pharmaceutical Research

Division.

Warner-Lambert Company,

Ann Arbor,

Michigan 48105-2430, USA

yielded twelve X-ray sources within a circle of radius $0.25^{\circ}$ centred on G10.1-0.3. Only one source of the twelve, at coordinates (1950) $18 \mathrm{~h} 0.5 \mathrm{~min}$ $41.56 \mathrm{~s},-20^{\circ} 25^{\prime} 3.4^{\prime \prime}$ (counting rate $1.4 \times 10^{-3} \pm 0.4 \times 10^{-3}$ counts $\mathrm{s}^{-1}$ in the energy range $0.22-2.5 \mathrm{keV}$ ) is within the revised localization box of SGR1806-20. The X-ray source is also coincident (within the estimated positional error of 11 arcs, which includes random and systematic errors) with the compact nebula of Kulkarni et al. ${ }^{5}$. The source extraction procedure presents no evidence of any extension of the X-ray source, but note that the Vela pulsar (at $500 \mathrm{pc}$ ) exhibits ${ }^{6}$ a wide range of angular scales $\left(<18^{\prime \prime}\right.$ to $\sim 1^{\circ}$ ) of pulsar-associated X-ray features. Such features might be seen in G10.1-0.3 with a longer observation or a more sensitive X-ray telescope.

An examination of the associated optical field reveals only one potential candidate for the X-ray source. This is a reddened object close to the IIIaJ plate limit and positioned on the edge of the $\mathrm{X}$-ray error box diagonally opposite the radio position reported in ref. 5 . A suitably obscured low-mass X-ray binary 